\title{
Interaction between dynamics and thermodynamics during tropical cyclogenesis
}

\author{
S. Gjorgjievska and D. J. Raymond \\ Physics Department and Geophysical Research Center, New Mexico Institute of Mining and Technology, Socorro, New \\ Mexico 87801, USA
}

Correspondence to: S. Gjorgjievska (saska@nmt.edu)

Received: 26 April 2013 - Published in Atmos. Chem. Phys. Discuss.: 16 July 2013

Revised: 25 January 2014 - Accepted: 17 February 2014 - Published: 27 March 2014

\begin{abstract}
Observational data of tropical disturbances are analyzed in order to investigate tropical cyclogenesis. Data from 37 cases observed during three field campaigns are used to investigate possible correlations between various dynamic and thermodynamic variables. The results show that a strong mid-level vortex is necessary to promote spin up of the lowlevel vortex in a tropical cyclone. This paper presents a theory on the mechanism of this process. The mid-level vortex creates a thermodynamic environment conducive to convection with a more bottom-heavy mass flux profile that exhibits a strong positive vertical gradient in a shallow layer near the surface. Mass continuity then implies that the strongest horizontal mass and vorticity convergence occurs near the surface. This results in low-level vortex intensification.

For two of the disturbances that were observed during several consecutive days, evolution of the dynamics and thermodynamics is documented. One of these disturbances, Karl, was observed in the period before it intensified into a tropical storm; the other one, Gaston, was observed after it unexpectedly decayed from a tropical storm to a tropical disturbance. A hypothesis on its decay is presented.
\end{abstract}

\section{Introduction}

The genesis phase is the least understood part of the tropical cyclone life cycle. In this paper we use the term cyclogenesis to refer to the intensification of a precursor disturbance into a tropical storm (TS). We know that tropical storms develop from preexisting finite amplitude disturbances, such as mesoscale convective systems (MCSs) that exhibit cyclonic vertical vorticity. They often form within monsoon troughs or tropical waves. Genesis involves processes that are much less important during the mature phase of a tropical cyclone. Favorable conditions for these MCSs to develop into tropical storms are high sea surface temperature and low vertical wind shear. These conditions are probably best summarized by Gray (1968). Although during a hurricane season many MCSs develop, only a small percentage of these turn into tropical storms, even in favorable environmental conditions. How are the developing disturbances different from the nondeveloping? McBride and Zehr (1981) compared composites of non-developing and developing cloud clusters. Their main findings were as follows: both systems have warm cores in the upper levels, but the warming is more pronounced in the developing clusters; the static stability is somewhat smaller for the developing systems; the developing composites exhibit less vertical wind shear near the system center, but there is a requirement for a certain kind of wind shear surrounding the developing system; and the developing clusters exhibit larger positive vorticity at low levels.

Over the last two decades a handful of field experiments were conducted. In situ data from tropical disturbances became available, which helped towards understanding the processes involved in tropical cyclogenesis, and theories of tropical storm formation have emerged. One question addressed by these theories is how a surface-intensified vortex develops with warm-core structure above the boundary layer; in particular, what aspects of the initial state are necessary to promote development of such a structure. Two features have been discussed in recent literature, namely, a mid-level vortex that precedes tropical cyclogenesis, and a surrounding kinematic boundary that protects the region of storm development from exterior influences above the boundary layer. In 
thermal wind balance, the precursor mid-level vortex corresponds to a cold-core vortex below the vorticity maximum and warm-core vortex aloft. Later the near-surface vorticity increases, a warm-core vortex develops in the lower troposphere, and genesis occurs. The available observations, though not abundant, show support for this pathway of development. This was the case with typhoon Robyn (1993) (Harr and Elsberry, 1996) and typhoon Nuri (2008) (Raymond and López, 2011) in the west Pacific and hurricane Guillermo (1991) in the east Pacific (Bister and Emanuel, 1997). There is also evidence of the pre-hurricane Dolly (1996) in the Atlantic developing a mid-level vortex $35 \mathrm{~h}$ before it was upgraded to a tropical depression (TD) (Reasor et al., 2005). This pathway of development was also observed in numerical simulations. Nolan (2007) used a model to simulate development of tropical cyclones, initiating each simulation with a preexisting vortex. All of his simulations, even the ones initiated with a low-level vortex, first developed a mid-level vortex before low-level spin up occurred. A "top-down" development, meaning a mid-level vortex developed as a precursor to cyclogenesis, was evident in these studies, but there was no physical explanation given of how the mid-level vortex encourages vorticity intensification near the surface. Later Raymond et al. (2011), based on the previous theoretical work by Raymond and Sessions (2007), hereafter RS07, and some observations, suggested that the thermodynamic state of the atmosphere within the mid-level vortex favors convection that aids the formation of the low-level vortex.

Meanwhile, Dunkerton et al. (2009) hypothesized that genesis is initiated within westward moving tropical waves, in a region near the intersection of the wave axis and the critical latitude. The latter is defined as the latitude at which the wave speed is the same as the background flow. They call this intersection a sweet spot, and the broader recirculating area around it is dubbed the pouch. The pouch exhibits low-level cyclonic vorticity and minimal strain rate. The air within the pouch is protected from lateral dry air intrusion, and thus continuous moist convection is enabled, which further increases the cyclonic vorticity. Ultimately, the waveconvection interaction leads to the development of rotating deep convection. Aggregation of low-level vorticity associated with the rotating deep convection results in a vortex intensification in the lower troposphere and, subsequently, in the middle and upper troposphere. The vortex eventually separates from the mother wave. This hypothesis, known as the marsupial paradigm, represents so-called "bottom-up" development.

The terms "top-down" and "bottom-up" give the impression of two opposed theories. Wang (2012) conducted numerical simulations of hurricane Felix (2007) and analyzed the genesis on two different scales, $2 \times 2$ degree box (mesobeta) and $6 \times 6$ degree box (meso-alpha), both centered on the pouch at $850 \mathrm{hPa}$. Her results show a "bottom-up" pathway of development on meso-beta scale, and a "top-down" pathway of development on meso-alpha scale. Dunkerton et al. (2009) also noted that the upward and downward pathways are not mutually exclusive and may co-exist on different scales. We believe that both the mid-level vortex (at meso-alpha) and the pouch (at meso-beta) are important for tropical cyclogenesis and they are perhaps two pieces of the same puzzle. In the future we should avoid the terms "topdown" and "bottom-up", as they are relative and are a source of confusion.

In the present paper we analyze data from developing and non-developing disturbances that were observed during the 2010 hurricane season in the tropical Atlantic and the Caribbean. For part of the analysis we also use data gathered in the west Pacific during the 2008 typhoon season. The results reinforce the importance of the mid-level vortex as a precursor to tropical storm formation.

There are two purposes to this paper. First, we present a theory on the mechanisms by which the mid-level vortex fosters low-level vortex spin up. Second, we hypothesize as to how an observed storm decayed. The remainder of this paper is organized as follows. The second section of this paper describes the data and the methods that are used to analyze the data. Section 3 gives the equations we use for our calculations. In Sect. 4 we give background on the theory. The results from our analysis are presented in Sect. 5. We discuss the results and give concluding notes in Sect. 6.

\section{Data and methods}

Three data sets are used for the purpose of our analysis. One set was gathered during the field campaign Pre-Depression Investigation of Cloud systems in the Tropics (PREDICT). The objectives and the tools of this campaign are described by Montgomery et al. (2012). The target areas of research were the North Atlantic and the Caribbean. The campaign took place during the period August-September of 2010 and was based on the island of St. Croix in the Caribbean. The main tools for gathering data were dropsondes. About twenty-five dropsondes per mission were launched from the National Science Foundation (NSF)-National Center for Atmospheric Research (NCAR) research aircraft, Gulfstream $\mathrm{V}(\mathrm{G}-\mathrm{V})$. The aircraft was also equipped with on-board instrumentation for measuring different meteorological parameters, but for our analyses we use the dropsonde data only. The launching altitude was $11-13 \mathrm{~km}$, so that data were recorded throughout almost the entire depth of the troposphere. Roughly, the dropsondes were distributed on a rectangular longitude-latitude grid, with grid spacing approximately $1^{\circ}$. The data were quality controlled and processed by NCAR's Earth Observing Laboratory (EOL). Twenty-six missions were conducted during which approximately 600 dropsondes were released. Eight disturbances were observed, for most of which multiple missions were flown. Four of these intensified into tropical storms, one of which became a category 3 hurricane. 
The second data set is from the NASA field campaign Genesis and Rapid Intensification Processes (GRIP), for which the time period and target area overlapped with PREDICT. The experiments were coordinated, and for several pre-storm disturbances observations were done by both GRIP and PREDICT. In the present study we use dropsonde data from six GRIP missions, for which dropsondes were launched from the NASA DC-8 aircraft. Braun et al. (2012) describe the tools and the objectives of the GRIP experiment.

The third data set was gathered during the field campaign Tropical Cyclone Structure (TCS08) experiment that took place in the period August-September 2008. The Naval Research Laboratory (NRL) P-3 aircraft and two Air Force Reserve WC-130 aircraft deployed dropsondes over the west Pacific. In addition, the P-3 aircraft took wind measurements using the ELectra DOppler RAdar (ELDORA). The quality control on the dropsonde data was also done by EOL. See Raymond and López (2011) and Raymond et al. (2011), hereafter RSL11, for more information.

Dropsondes measure temperature, pressure, horizontal wind and relative humidity continuously as they descend, so that the vertical resolution of the collected data is good. However, the horizontal resolution is coarse $\left(\sim 1^{\circ} \times 1^{\circ}\right)$. We further use a three-dimensional variational (3-D-Var) analysis to obtain values of the meteorological parameters in the entire observational volume. For each mission we first vertically interpolate the data from each dropsonde, so that they all have the same vertical interval and step size. We then also calculate the mixing ratio, moist entropy, saturation mixing ratio, and saturation moist entropy at every grid point. The dropsondes are not launched at the same time but rather one at a time, roughly one dropsonde every 7 $10 \mathrm{~min}$. Depending on the size of the disturbance, it takes $2-4 \mathrm{~h}$ for all the dropsondes to be released. For the purpose of our analyses we adjust dropsonde positions in the moving frame of the disturbance to their locations at a standard reference time. The moving frame is defined as a frame moving with the propagation velocity of the disturbance. The propagation velocity is calculated from the final analysis (FNL) for the Global Forecasting System by tracking the vorticity center averaged between $850-700 \mathrm{hPa}$. The standard reference time is usually chosen to be roughly half way through the period of dropsonde deployment, resulting in a snapshot of the disturbance at this time. The snapshot assumption is adequate for studying mesoscale processes. Then we specify a three-dimensional grid, moving with the observed disturbance, with longitude-latitude resolution $0.125^{\circ} \times 0.125^{\circ}$ and vertical resolution of $0.625 \mathrm{~km}$. The dropsonde data are assigned to appropriate grid points. The data are then interpolated to the full grid using the 3-D-Var scheme. The vertical velocity is calculated by strongly enforcing mass continuity in the 3-D-Var scheme. The 3-D-Var analysis also imposes a certain degree of smoothing in order to remove noise and aliasing. Finally, we mask the file in order to consider only the area covered by the dropsondes. The 3-D file at this point is ready for analysis.

A detailed description of the 3-D-Var technique used in the present work is given by López and Raymond (2011) and Raymond and López (2011). The values of the 3-D-Var parameters used here are the same as in RSL11.

\section{Equations}

\subsection{Vorticity budget equation}

We adopt the flux form of the vorticity budget equation from Raymond and López (2011):

$$
\frac{\partial \zeta_{z}}{\partial t}=-\nabla_{h} \cdot\left(\zeta_{z} \boldsymbol{v}_{h}-\zeta_{h} v_{z}+\hat{k} \times \boldsymbol{F}\right)-\hat{\boldsymbol{k}} \cdot \nabla_{h} \theta \times \nabla_{h} \Pi .
$$

Here, $\boldsymbol{v}=\left(\boldsymbol{v}_{h}, v_{z}\right)$ is the storm-relative velocity, $\zeta=\left(\zeta_{h}, \zeta_{z}\right)$ is the absolute vorticity, $\boldsymbol{F}$ is a force due to surface friction, $\theta$ is the potential temperature, and $\hat{\boldsymbol{k}}$ is the unit vector in the vertical direction. The quantity $\Pi=C_{\mathrm{p}}\left(p / p_{\text {ref }}\right)^{R / C_{\mathrm{p}}}$ is the Exner function, where $C_{\mathrm{p}}$ is the specific heat at constant pressure, $R$ is the gas constant for dry air and $p_{\text {ref }}=1000 \mathrm{hPa}$ is a constant reference pressure. The subscripts $h$ and $z$ denote the horizontal and vertical components, respectively. The last term on the right hand side represents the vertical component of the baroclinic generation of vorticity. For environments that are characterized by weak baroclinicity, as in the tropics, this term is insignificant and thus can be neglected. The expression in parentheses is the horizontal flux of $\zeta_{z}$ : the first term represents the flux due to advection, the second is the flux due to tilting, and the third is the flux due to the frictional force. The frictional force per unit mass, $\boldsymbol{F}$, is estimated by

$\boldsymbol{F}=\boldsymbol{\tau} \frac{\exp \left(-z / z_{\mathrm{s}}\right)}{\rho z_{\mathrm{s}}}$,

where $z$ is the height, $z_{\mathrm{s}}$ is a scale height which represents the average depth of the boundary layer, and

$\boldsymbol{\tau}=-\rho_{\mathrm{bl}} C_{\mathrm{D}}\left|\boldsymbol{U}_{\mathrm{bl}}\right| \boldsymbol{U}_{\mathrm{bl}}$

is the surface stress. The symbol $\rho$ represents the density, $\boldsymbol{U}_{\mathrm{bl}}$ is the horizontal wind, and $C_{\mathrm{D}}$ is a drag coefficient, which we calculate using the bulk formula

$C_{\mathrm{D}}=\left(1+0.028\left|\boldsymbol{U}_{\mathrm{bl}}\right|\right) \times 10^{-3}$.

Here $\boldsymbol{U}_{\mathrm{bl}}$ has units of meters per second. This is the same formula for the drag coefficient as used in RSL11.

\subsubsection{Thermodynamic variables}

Two important parameters that we analyze are the saturation fraction and the instability index. The saturation fraction is defined as the vertically integrated precipitable water divided 
by the vertically integrated saturated precipitable water,

$\mathrm{SF}=\frac{\int_{0}^{h} \rho r d z}{\int_{0}^{h} \rho r^{*} d z}$,

where $r^{*}$ is the saturation mixing ratio. $h$ is the height of the domain. Bretherton et al. (2004) found that this parameter controls most of the variability in rainfall over the tropical oceans. The instability index is a measure of the tropospheric instability to moist convection and it is defined as

$\Delta s^{*}=s_{\text {low }}^{*}-s_{\text {high }}^{*}$

Here $s_{\text {low }}^{*}$ is the saturated moist entropy averaged over the layer between 1 and $3 \mathrm{~km}$ and $s_{\text {high }}^{*}$ is the saturated moist entropy averaged over the layer between 5 and $7 \mathrm{~km}$. As the saturated moist entropy is a proxy for temperature at a fixed pressure level, larger values of this parameter are associated with an atmosphere more unstable to moist convection.

\section{Background}

It has long been known that both dynamics and thermodynamics are involved in the process of tropical storm formation. Dynamics can affect the thermodynamic processes and vice versa, so there is feedback between the two. Hence, in order to understand tropical cyclogenesis one should not only understand both the dynamics and thermodynamics, but should also understand how they work together and how they interact constructively to create a tropical storm. Observations of pre-storm disturbances over the last two decades have revealed that a mid-level vortex forms prior to tropical storm genesis. A low-level mesoscale vortex may or may not exist prior to a mesoscale mid-level vortex development, however, development/intensification of a low-level cyclonic vortex into a TS follows after an establishment of a mesoscale mid-level vortex. The Haynes and McIntyre (1987) flux form of the the vertical vorticity equation does not recognize direct communication between vortices at different levels, as it considers the dynamics alone. The theory we present here explains how the mid-level vortex influences the low-level vortex by changing the thermodynamic stratification of the troposphere. In thermal wind balance, a midlevel vortex is associated with a cold-core vortex below it and with a warm-core vortex above it.

To test the effect of the temperature perturbations associated with a mid-level vortex, RS07 did an experiment using a cumulus ensemble numerical model. The model was nonrotational, with interactive radiation. The simulations were done on a two-dimensional domain. First, they ran the model to radiative-moist-convective equilibrium (RCE). Then they used the RCE vertical profile of potential temperature and the corresponding vertical profile of moisture as reference profiles for weak temperature gradient (WTG) simulations. Two sets of WTG simulations were done. One with perturbed temperature profile, while the moisture profile remained unaltered, and another set with perturbed moisture profile, while the temperature profile remained the same. Control runs were done with unchanged reference profiles. The experiments regarding added moisture to the numerical troposphere with unaltered stability to moist convection resulted in more rainfall per unit surface entropy flux, while the resulting vertical mass flux profiles did not change their shape with respect to the control run. They only increased in magnitude. The other set of experiments, with unaltered moisture but changed stability to moist convection behaved differently. Stabilization of the troposphere (negative potential temperature perturbations applied below $5 \mathrm{~km}$ and positive perturbations above $5 \mathrm{~km}$ ) resulted in increased rainfall, but it also produced a differently shaped vertical mass flux profile from the control run. Its maximum occurred at a lower elevation (more bottom-heavy mass flux) and it had a larger magnitude. In other words, it exhibited a strong positive vertical gradient in a shallower layer near the surface. Such a mass flux would produce more low-level vorticity convergence and consequently low-level spin up. Raymond et al. (2011) analyzed observational data of seven tropical disturbances and found support for the theoretical results of RS07.

Raymond and Sessions (2007) did not discuss the mechanisms by which a more stable troposphere produces a stronger and more bottom-heavy mass flux profile. We did additional diagnostics of their simulations and found that the parcel buoyancy and the surface entropy fluxes are responsible for the observed changes in the mass flux profile. These are affected by the applied temperature perturbations (cooling below $5 \mathrm{~km}$ and warming above $5 \mathrm{~km}$ ) in the following way. The parcel buoyancy above $\sim 2 \mathrm{~km}$ is reduced, with larger reductions occurring at higher elevations. Consequently, the parcels encounter the level of neutral buoyancy at a lower elevation. The parcel buoyancy below $2 \mathrm{~km}$ is slightly increased, which corresponds to smaller convective inhibition (CIN). The surface entropy fluxes are larger in the perturbed cases, as a result of the lowered atmospheric temperature near the sea surface. We conclude that a more stable troposphere produces a more bottom-heavy vertical mass flux profile as a result of the corresponding changes in the parcel buoyancy. The larger mass flux magnitude is fostered by the larger surface entropy fluxes.

Smith and Montgomery (2012) and Montgomery and Smith (2012) questioned the validity of the RS07 theoretical study, because it was conducted within a non-rotating model. Introducing rotation might change the results significantly if the timescales of convection and vorticity were comparable. In the current study we are looking mainly at pre-storm disturbances, where the timescale for vorticity is several times larger then the timescale for convection. The time it takes a convective cell to travel from the boundary layer to the tropopause is about an hour, whereas the largest calculated area-averaged vorticity is $\sim 0.08 \mathrm{ks}^{-1}$, which corresponds to 
a timescale of $3.5 \mathrm{~h}$. Wissmeier and Smith (2011) did a study on this subject. They conducted idealized numerical experiments, to explore the effects of ambient vertical vorticity on convective updrafts, focusing on convection that develops within tropical depressions and pre-depressions. For the types of rotation they studied, they did not find the rotation to have large effects on the updraft dynamics. Therefore, we believe that introducing rotation would not change the results enough to affect the conclusions of the current work.

\section{Results}

The results are divided into two parts. The first part employs the 7 observational cases from Raymond et al. (2011) and 30 additional cases from PREDICT and GRIP, to further explore possible correlations between dynamic and thermodynamic variables. The second part presents the evolution of two disturbances that were observed during PREDICT and compares the two. One of those, Gaston, had a tropical storm status for a few hours only, after which it started to decay. Nevertheless, it was observed over the course of the next 5 days, as it was expected to intensify again. However, it kept decaying slowly until it dissipated and we take it as a decaying/non-developing case. The other is Karl, a developing system that eventually became a major hurricane. It was observed extensively in its pre-storm phase.

Many of the results shown in this paper are obtained by horizontal averaging over an area for each disturbance. A valid question arises here, regarding the proper area to be considered for analysis. Unfortunately, there does not yet exist an established, objective method for quantitatively comparing the observed disturbances. It is difficult to find such a method, because of inconsistencies among disturbances. There are two main factors driving these inconsistencies. First, there are differences in aircraft data sampling. The observational areas have different sizes and shapes, and the circulation centers are in different locations within the observational area. The other source of inconsistency which is probably more important but is often neglected, is the uniqueness of each disturbance, regardless of the data sampling area. No two disturbances have the same size, shape, and structure, nor do they occur within identical environments. The pre-storm disturbances in particular are highly non-axisymmetric. Therefore, whatever method is chosen to select an area for analysis will skew the results in some way. For example, one may choose a circular or a rectangular area centered on a circulation center at a certain level for all the disturbances. The size of the area may cover $50 \%$ of the actual disturbance size for some disturbances, and for some may cover the entire disturbance. Furthermore, in the case of a tilted vortex axis, the results will be skewed in favor of the level for which the circulation center is defined as being the center of the analysis domain. Thus, either choosing the same area, size, and shape for all the disturbances, or sub- jectively choosing each area for analysis, based on moisture, convective mass flux, and rotation parameters, can each skew the results to some degree.

Keeping the above in mind, we obtained two sets of results. For the first set, averaging was done over a subjectively chosen area for analysis. The criteria were to cover as much of the disturbance as possible, encompass all the convective activity and the high saturation fraction regions, and center the area on the $5 \mathrm{~km}$ circulation center if possible, without significantly compromising the previous two criteria. For the second set, the analysis was repeated by averaging over the entire observational area, minus the area that was obviously not part of the disturbance. The conclusions of this paper are invariant to the differences between these two sets of results.

To avoid confusion, here we clarify the terminology used throughout the paper. The term mid-level vortex refers to a vortex located anywhere between 3 and $6 \mathrm{~km}$, and the significance of this vortex is its association with a cold core beneath it and a warm core above it. In other words, there is a positive vertical gradient of relative vorticity in a deep layer adjacent to the surface. Whether the maximum vorticity occurs at 4 , 5 , or $6 \mathrm{~km}$ does not affect the interpretation of our findings. With the term lower levels we usually refer to the levels below $3 \mathrm{~km}$, adjacent to the surface, and the term upper levels refers to levels above $5 \mathrm{~km}$. A mass flux profile that exhibits the strongest vertical gradient in the lowest $2-3 \mathrm{~km}$ is called bottom-heavy.

\subsection{Correlation between dynamics and thermodynamics}

The scatter plots presented in this section consist of data points from 37 cases: 24 from PREDICT, 6 from GRIP and 7 from TCS08. Table 1 shows the date, propagation velocity, reference time, area-averaged Reynolds sea surface temperature (SST), and National Hurricane Center (NHC) classification for each mission conducted into these disturbances. All but two cases observed during PREDICT are included in the scatter plots. The test run from PREDICT has not been entered because the dropsonde pattern did not enclose any area, and the third mission in Fiona is not considered, as its circulation was strongly influenced by hurricane Earl. Each data point represents a single mission into a disturbance of interest.

Three scatter plots are shown in Fig. 1. Each case in the scatter plots has its own unique characteristics (size, shape, environment). For this reason the plots are noisy, but there is a definite significant trend in all of them. The conclusions of this paper rest partly on these trends. Figure 1a plots the instability index versus the mid-level relative vorticity. The mid-level vorticity is calculated as an area average at $5 \mathrm{~km}$ elevation. There is nothing special about this level. We simply choose it as a representative of the mid-level vorticity in all the disturbances, regardless of where the maximum vorticity occurs in each separate case. The trend in the scatter 
Table 1. Observational cases that enter the scatter plots in Sect. 5.1. The stage of each disturbance is as recognized by NHC at the time of observation. "Low" stands for either a tropical wave or a weak tropical disturbance, while TD indicates a tropical depression and TS a tropical storm.

\begin{tabular}{|c|c|c|c|c|c|}
\hline & Date & Ref. time (UTC) & $\boldsymbol{v}_{\mathrm{p}}\left[\mathrm{ms}^{-1}\right]$ & $\operatorname{SST}\left[{ }^{\circ} \mathrm{C}\right]$ & Stage \\
\hline PGI27-1 & $17 / 08 / 2010$ & $12: 00$ & $(-8.0,0.0)$ & 29.8 & low \\
\hline PGI27-2 & $18 / 08 / 2010$ & $14: 30$ & $(-7.0,0.0)$ & 29.6 & low \\
\hline PGI30-1 & $21 / 08 / 2010$ & $14: 00$ & $(-8.0,0.0)$ & 28.0 & not classified \\
\hline PGI30-2 & $23 / 08 / 2010$ & $12: 00$ & $(-7.0,2.5)$ & 29.4 & not classified \\
\hline Fiona 1 & $30 / 08 / 2010$ & 13:00 & $(-8.9,0.0)$ & 28.5 & low \\
\hline Fiona 2 & $31 / 08 / 2010$ & 13:00 & $(-10.1,3.7)$ & 29.3 & $\mathrm{TD}$ \\
\hline Gaston 1 & $02 / 09 / 2010$ & $17: 00$ & $(-3.0,1.0)$ & 28.2 & TD \\
\hline Gaston 2 & 03/09/2010 & $16: 00$ & $(-3.1,1.8)$ & 28.4 & low \\
\hline Gaston 3 & $05 / 09 / 2010$ & $16: 00$ & $(-6.7,0.0)$ & 28.7 & low \\
\hline Gaston 4 & $06 / 09 / 2010$ & $14: 00$ & $(-6.7,0.0)$ & 29.1 & low \\
\hline Gaston $4 \mathrm{a}$ & $06 / 09 / 2010$ & $21: 30$ & $(-9.25,-0.5)$ & 29.3 & low \\
\hline Gaston 5 & $07 / 09 / 2010$ & $14: 00$ & $(-6.7,0.0)$ & 29.4 & low \\
\hline Gaston 5a & $07 / 09 / 2010$ & $21: 30$ & $(-8.7,1.0)$ & 29.4 & low \\
\hline Karl 1 & $10 / 09 / 2010$ & $11: 15$ & $(-5.1,0.0)$ & 30.0 & not classified \\
\hline Karl 2 & $10 / 09 / 2010$ & $19: 00$ & $(-2.0,0.9)$ & 30.0 & not classified \\
\hline Karl 3 & $11 / 09 / 2010$ & $17: 45$ & $(-6.0,0.0)$ & 30.2 & low \\
\hline Karl 4 & $12 / 09 / 2010$ & $13: 30$ & $(-6.8,0.0)$ & 30.0 & low \\
\hline Karl 4a & $12 / 09 / 2010$ & $21: 20$ & $(-6.7,1.5)$ & 30.0 & low \\
\hline Karl 5 & $13 / 09 / 2010$ & $13: 45$ & $(-6.1,2.8)$ & 30.1 & low \\
\hline Karl 5a & $13 / 09 / 2010$ & $22: 30$ & $(-6.7,1.5)$ & 30.1 & low \\
\hline Karl 6 & $14 / 09 / 2010$ & $17: 00$ & $(-6.1,2.8)$ & 30.0 & TS \\
\hline Matthew 1 & $20 / 09 / 2010$ & $15: 00$ & $(-5.1,-1.5)$ & 29.9 & low \\
\hline Matthew 2 & $21 / 09 / 2010$ & $14: 30$ & $(-6.0,0.0)$ & 30.1 & low \\
\hline Matthew 2a & $21 / 09 / 2010$ & $20: 10$ & $(-6.0,0.0)$ & 30.0 & low \\
\hline Matthew 3 & $22 / 09 / 2010$ & $16: 00$ & $(-6.0,0.0)$ & 29.8 & low \\
\hline Matthew 3a & $22 / 09 / 2010$ & $19: 10$ & $(-6.0,0.0)$ & 29.7 & low \\
\hline Matthew 4 & $24 / 09 / 2010$ & $16: 00$ & $(-8.9,0.0)$ & 29.7 & TS \\
\hline Nicole 1 & $27 / 09 / 2010$ & $16: 00$ & $(0.0,0.0)$ & 29.6 & low \\
\hline Nicole 2 & $28 / 09 / 2010$ & $16: 00$ & $(1.5,4.2)$ & 29.6 & TS \\
\hline PGI48/50 & $30 / 09 / 2010$ & $15: 15$ & $(-6.2,2.3)$ & 29.5 & low \\
\hline Nuri 1 & $15 / 08 / 2008$ & $25: 50$ & $(-7.0,0.0)$ & 29.8 & low \\
\hline Nuri 2 & $16 / 08 / 2008$ & $23: 55$ & $(-8.7,0.0)$ & 29.9 & $\mathrm{TD}$ \\
\hline TCS025-1 & $27 / 08 / 2008$ & 00:00 & $(2.4,2.6)$ & 29.5 & low \\
\hline TCS025-2 & $28 / 08 / 2008$ & 00:00 & $(2.4,2.6)$ & 29.2 & low \\
\hline TCS030 & 01/09/2008 & $24: 00$ & $(-6.3,0.6)$ & 30.1 & low \\
\hline TCS037 & 07/09/2008 & $21: 05$ & $(-5.7,3.2)$ & 28.2 & low \\
\hline Hagupit 2 & $14 / 09 / 2008$ & $23: 35$ & $(-2.3,1.1)$ & 29.9 & low \\
\hline
\end{tabular}

plot suggests that a smaller instability index corresponds to a stronger mid-level vorticity. This result is not surprising because it is in agreement with thermal wind balance: mid-level vorticity is associated with a cooler lower troposphere and/or a warmer upper troposphere, which translates into stabilization of the atmosphere, that is, a decrease of the instability index.

Figure $1 b$ is a scatter plot of instability index versus saturation fraction. It demonstrates negative correlation between these two variables. This tells us that a more stable troposphere tends to be moister. Figure 1c shows a scatter plot between the instability index and the low-level vorticity ten- dency. The latter is calculated as an average over the lowest kilometer. The negative slope is obvious, which suggests that larger vorticity tendencies are associated with a more stable troposphere. Figure $1 \mathrm{~b}$ and $\mathrm{c}$ suggest that the thermodynamics associated with strong mid-level vorticity are conducive to the increase of the near-surface vorticity.

All the scatter plots from Fig. 1 were recreated by calculating the mid-level vorticity as an average between 3 and $5 \mathrm{~km}$, and the low-level vorticity tendency as an average between 0 and $2 \mathrm{~km}$. The trends were very similar to the ones presented here, indicating that the results are insensitive to the precise averaging areas. 
(a)

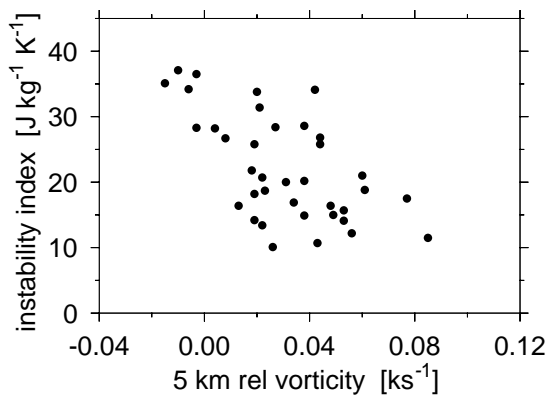

(b)

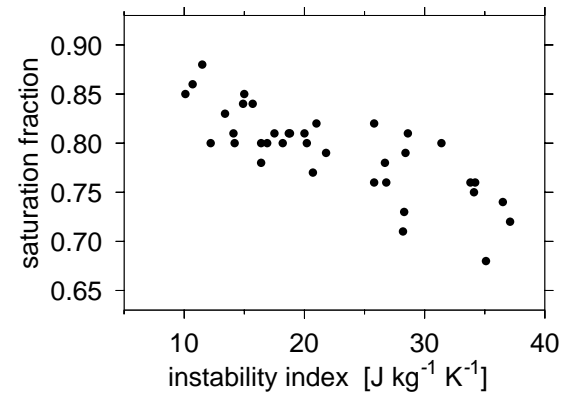

(c)

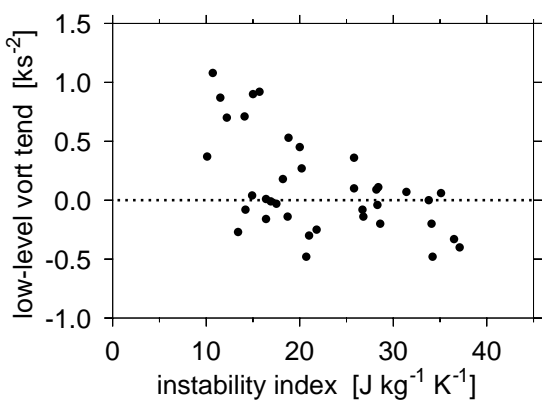

Fig. 1. Scatter plots of (a) mid-level relative vorticity versus instability index, (b) instability index versus saturation fraction, and (c) instability index versus low-level vorticity tendency.

Table 2. Statistics on the scatter plots in Fig. 1.

\begin{tabular}{lcc}
\hline Variables & Correlation coeff. & $p$ value \\
\hline Mid-level vorticity vs. instability index & 0.32 & 0.04 \\
Instability index vs. saturation fraction & 0.60 & 0.0002 \\
\hline Instability index vs. low-level vorticity tendency & 0.35 & 0.05 \\
\hline
\end{tabular}

We do not expect strict correlations between the analyzed variables. It is not even clear if the possible correlations that are implied in the scatter plots are linear or not. The conclusions of our analysis are based solely on the trends that are obvious in the scatter plots presented, positive or negative. Keeping this in mind, one might wonder how strong these correlations are. Assuming linear correlations between all the variables, we conducted statistical analysis on these data. The correlation coefficients and the $p$ values are given in Table 2. The significance level is greater than $95 \%$ for all the correlations. The statistics for the analogous scatter plots from the other set of results, where minimal area selection was done, show similar numbers. Therefore, the observed trends in the scatter plots are robust and they strongly support the idea of the mid-level vortex promoting near-surface vorticity intensification via a change in the tropospheric thermodynamic stratification on the mesoscale. It is true that cor- relation does not imply causation, but the results of Raymond (2012) strongly imply that the arrow of causality points from the mid-level vortex to the thermodynamics, and not vice versa.

\subsection{Karl and Gaston}

We now analyze the evolution of the vertical profiles of vorticity tendency, vertical vorticity, vertical mass flux, moist entropy and saturated moist entropy for Karl and Gaston. The vertical profiles of the analyzed variables represent horizontal averages over the respective areas selected for analysis.

\subsubsection{Karl}

Karl started as a disturbance within the Intertropical Convergence Zone (ITCZ), just northeast of the South American 
(a)

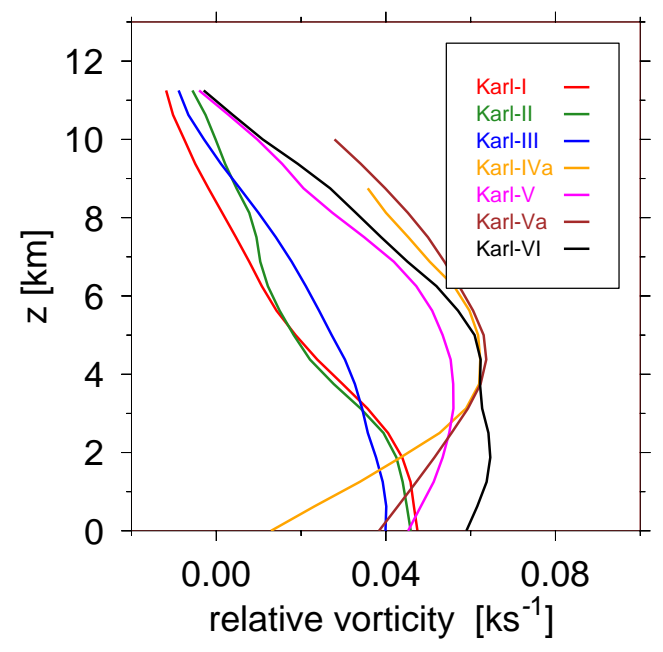

(c)

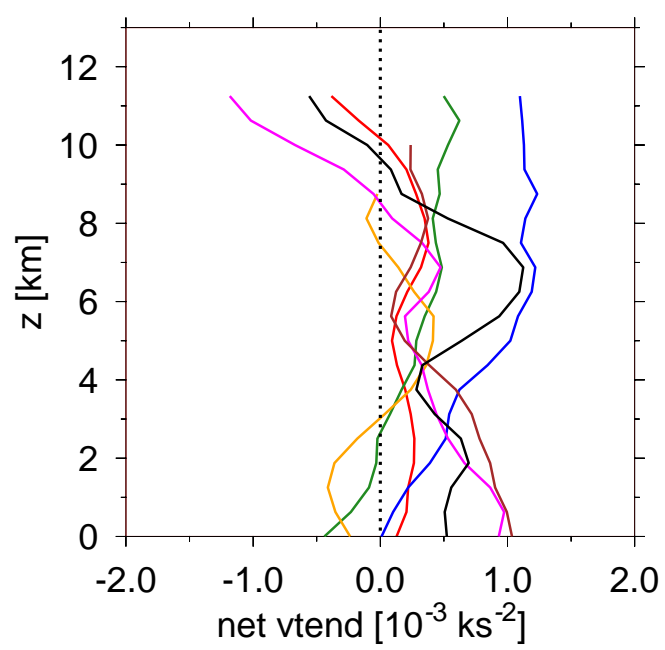

(b)

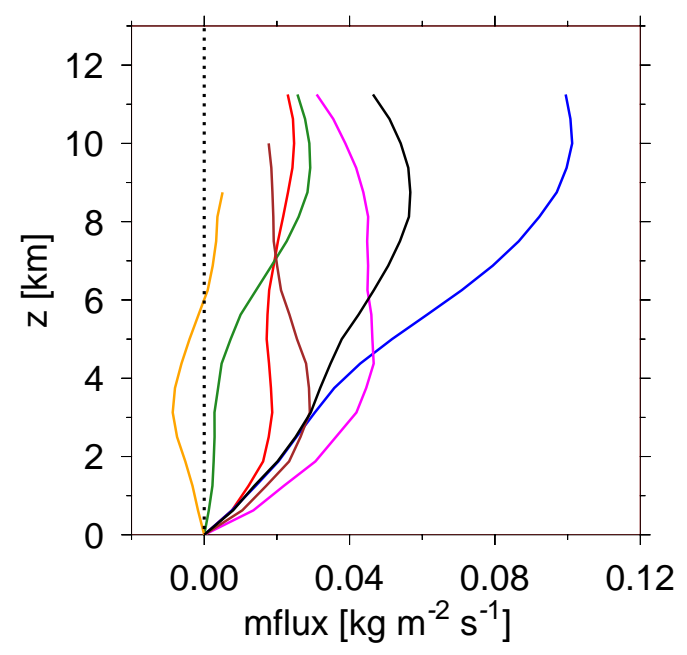

(d)

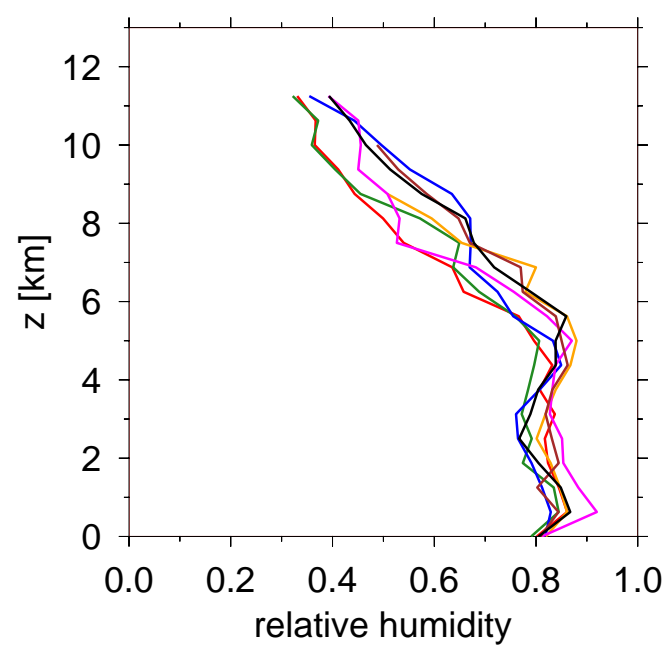

Fig. 2. Horizontally averaged (a) relative vertical vorticity, (b) vertical mass flux, (c) vorticity tendency, and (d) relative humidity for all the Karl missions.

coast. It was a broad low pressure area with active convection. Two observations in this disturbance were conducted before the NHC declared it an area of interest. Thus, the genesis phase of Karl, which ultimately developed into a category 3 hurricane, was well captured.

Because of airspace restrictions, the fourth PREDICT research flight did not cover an adequate observational area (the circulation center was not even partly covered) and, therefore, is excluded from the evolution analysis. The results from the other five PREDICT missions and two GRIP missions are summarized in Fig. 2. Karl 1 was conducted on 10 September. The disturbance exhibited weak cyclonic vor- ticity with a SW tilt of the vorticity axis (not shown). The largest vorticity was observed in the lowest kilometer, as the red line in Fig. 2a shows. The mass flux profile reflects the large convective activity that was occurring at that time. The convective flux was bottom heavy, which suggests that extensive stratiform regions did not exist at that time. The entire column was moist (Fig. 2d). The vorticity tendency was overall positive, though small in magnitude (Fig. 2c). Several hours later, the Karl 2 mission was conducted (the reference times of the two missions were about $9 \mathrm{~h}$ apart). The most notable change was in the mass flux profile which was now top heavy (Fig. 2b, green line), suggesting dominance 
(a) Karl

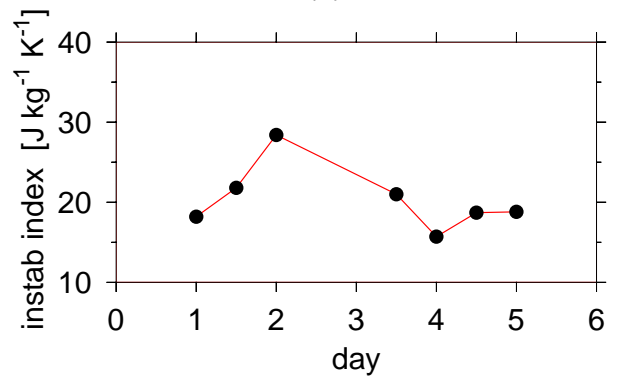

(b) Karl

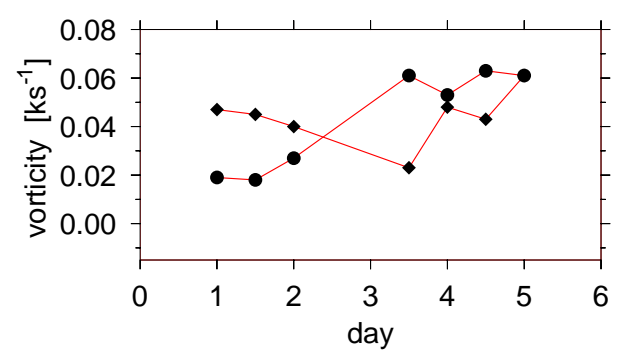

(c) Gaston

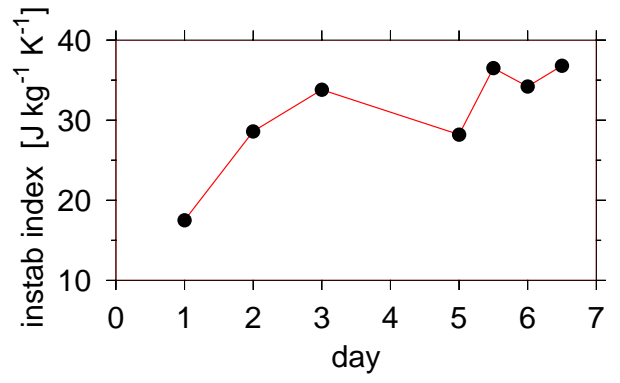

(d) Gaston

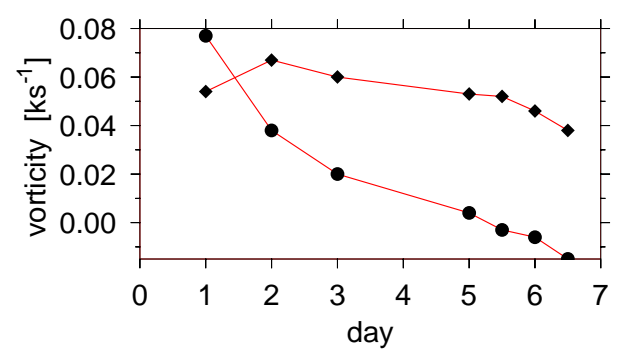

Fig. 3. Horizontally averaged (a) relative vertical vorticity, (b) vertical mass flux, (c) vorticity tendency, and (d) relative humidity for all the Gaston missions.

of stratiform clouds, which perhaps resulted as further development of the convective clouds from earlier that day. The green line in Fig. $2 \mathrm{~d}$ shows that the relative humidity also decreased in the layer between 1 and $5 \mathrm{~km}$, which is also consistent with the lack of convective clouds. The relative vorticity stayed virtually the same, but the vorticity tendency was negative in the lowest level (green lines in Fig. 2a and c). The vorticity axis maintained a SW tilt.

Bursts of strong convection to the east of the circulation center were observed on 11 September, when the G-V conducted its third mission into Karl. The blue lines in Fig. 2 refer to Karl 3. The vertical profile of the relative vorticity shows a slight decrease at low levels and an increase at midlevels. The vertical mass flux was top-heavy and strong, with a peak near $10 \mathrm{~km}$, resulting in the large positive values of the vorticity tendency at mid-levels. At this point Karl became an area of interest to the NHC. The large mid-level vorticity tendency from Karl 3 resulted in significant mid-level vorticity increase, which was observed during the GRIP mission that was conducted on the evening of 12 September (Fig. 2a, orange line). On 13 September, the G-V conducted the fifth mission into the disturbance, which at that time was located over the warm waters of the northwestern Caribbean Sea. At this point Karl had redeveloped the low-level vortex (purple lines in Fig. 2). The relative humidity had increased in the lowest $3 \mathrm{~km}$. Convection covered a large fractional area of the observational region. Deep convective and stratiform clouds were observed. The vertical mass flux profile was bottomheavy, reflecting dominance of convective clouds. An associated large positive vorticity tendency was registered at low levels. The vortex axis was vertical by that time (not shown). A similar situation was observed later that same day (Fig. 2, brown lines). The last PREDICT mission into Karl was conducted the following day, when significant low-level vorticity increase was observed (Fig. 2, black line). By the time the G$\mathrm{V}$ returned from this mission, NHC had upgraded Karl to a tropical storm.

Karl kept intensifying; on 16 September, it reached hurricane strength, and one day later it became a major hurricane. It made a landfall on the Yucatán peninsula, Mexico, and caused significant damage.

In summary, Karl was another example where the development of a mid-level vortex preceded genesis. It started as a weak vortex in a sheared, moist environment, but the near surface vortex did not intensify until after the mid-level vortex developed. The observed evolution of Karl from a tropical disturbance to a tropical storm supports the proposed theory of the role of the mid-level vortex in low-level vortex intensification. Fig. 4 a and b show the time series of the instability index and the relative vorticity. The instability index on day 
(a)

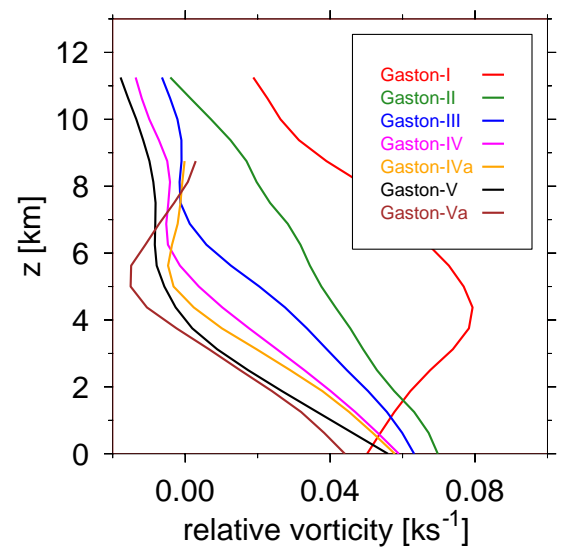

(c)

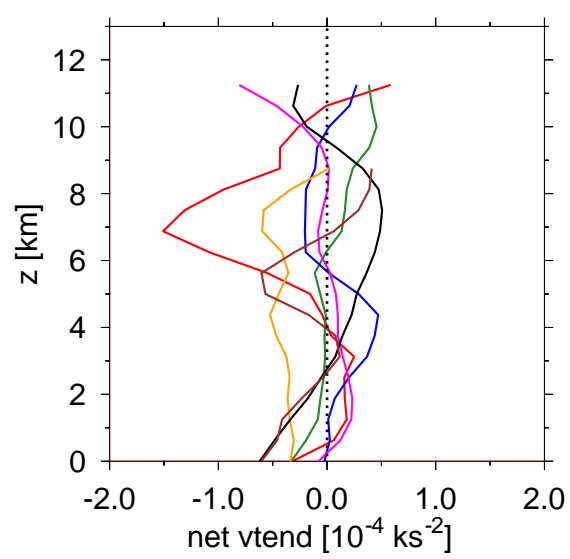

(b)

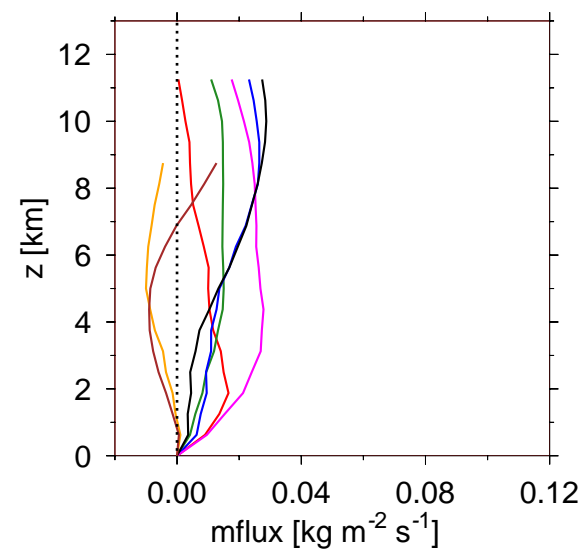

(d)

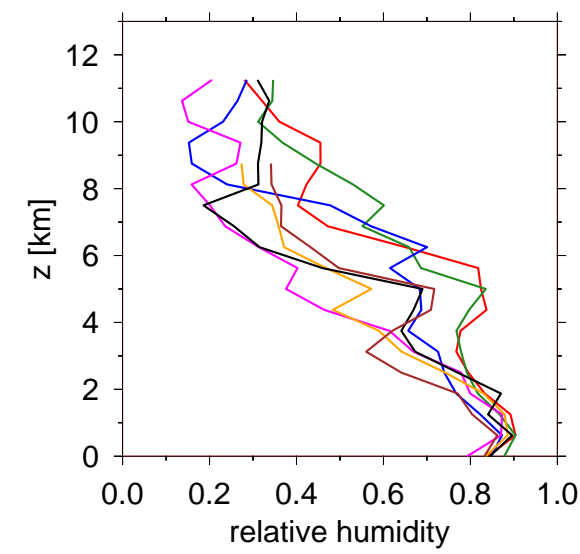

Fig. 4. Karl (left column) and Gaston (right column). The upper panels show the time series of the instability index. The solid circles in the lower panels are for the mid-level relative vorticity and the diamonds are for the low-level relative vorticity.

1 of observation was low, but it increased between the first two missions into Karl. During that time, the mid-level vortex was not well developed and it was weaker than the lowlevel vortex. By the third mission the mid-level vorticity had slightly increased, but most importantly, the instability index had further increased. A corresponding strong, top-heavy mass flux profile was observed (Fig. 2b, blue line), which is in agreement with the theoretical results of Raymond and Sessions (2007). As a response to this mass flux profile of Karl 3, the following day significant mid-level vortex intensification was observed, as was a corresponding decrease of the instability index. From here, the low-level vortex started intensifying as well, and in less than $48 \mathrm{~h}$ Karl became a tropical storm.

\subsubsection{Gaston}

Gaston emerged from Africa as an MCS embedded in an easterly wave. Seven missions were conducted in this disturbance over the course of six days. On its first mission in this disturbance, the G-V reached Gaston as it was being downgraded from a tropical storm to a tropical depression. Shortly afterward, it was further downgraded to an area of interest. Figure 3 shows the vertical profiles of area-averaged relative vorticity, vertical mass flux, vorticity tendency, and relative humidity. The red lines correspond to Gaston 1 . The relative vorticity at this time had a maximum at $4-5 \mathrm{~km}$. Convection occurred in a small region near the $5 \mathrm{~km}$ circulation center and it was surrounded by downdrafts. This explains the small, area-averaged vertical mass flux magnitude (Fig. 3b). Negative vorticity tendency was observed in the middle levels. 
(a) Gaston 1: $5 \mathrm{~km}$

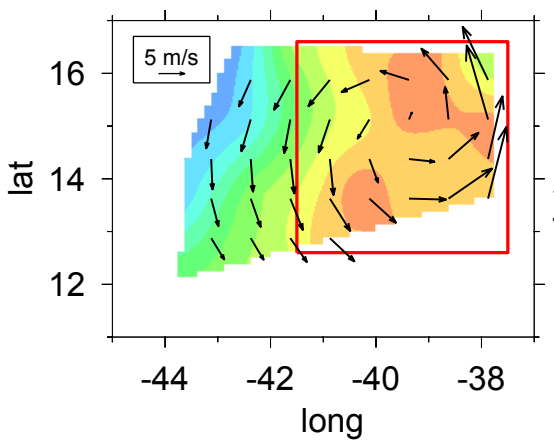

(c) Gaston 1: 7 km

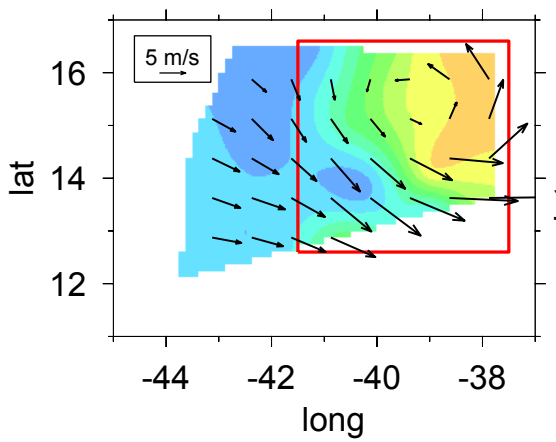

(b) Gaston 2: 5 km

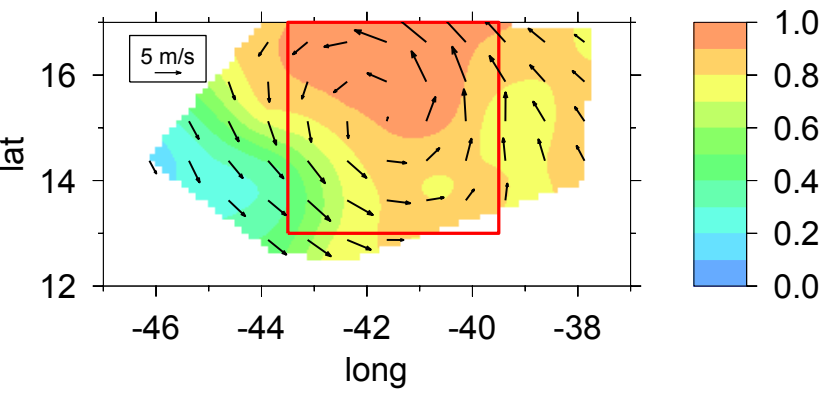

(d) Gaston 2: $7 \mathrm{~km}$

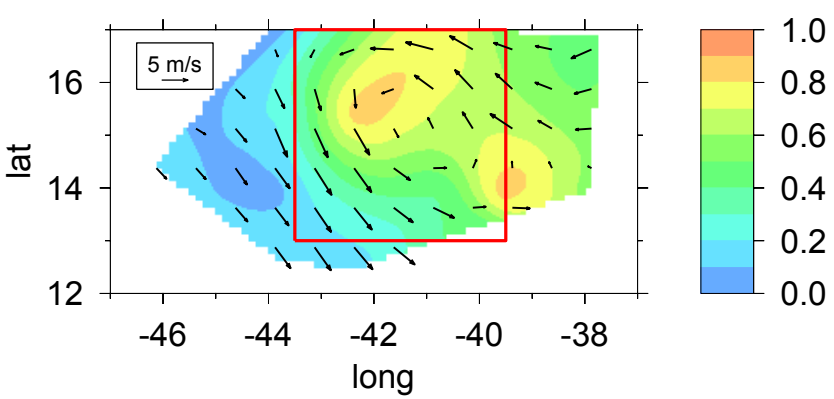

Fig. 5. Relative humidity during Gaston 1 (left panels) and Gaston 2 (right panels) at $5 \mathrm{~km}$ and at $7 \mathrm{~km}$. The vectors represent the relative wind at the respective levels. The red boxes enclose the area selected for analysis.

Gaston 2 was conducted the following day. The green lines in Fig. 3 show the area-averaged vertical structure of Gaston 2 . The most obvious feature is that the relative vorticity changed drastically from the previous day, even though a vortex was still evident (Fig. 5b). The low-level vorticity, however, was greater, in spite of the negative vorticity tendency from the previous day. This disagreement is most likely due to the fact that the vorticity tendency equation only provides a snapshot at a particular time. The vertical mass flux profile at this time exhibited a maximum near $4 \mathrm{~km}$, but the convective regions covered a smaller fractional area of the disturbance than the downdrafts, so that the magnitude of area-averaged vertical mass flux had not changed from the previous day. The area-averaged relative humidity remained almost the same. By the next day (Gaston 3), the relative vorticity decreased at all levels and also the relative humidity decreased notably (Fig. 3a, d; blue lines). The rest of the missions conducted in this disturbance revealed a further trend of decrease in relative vorticity and relative humidity.

Figure 4 summarizes the evolution of the thermodynamics and dynamics for Gaston during the period it was observed. At time 1, Gaston featured a low instability index and a strong mid-level vortex. According to our theory, this is favorable for low-level vorticity increase, which did indeed happen by time 2 . However, in the period between 1 and 2 , a significant decrease in mid-level vorticity and a cor- responding increase in the instability index occurred. After time 2 , the relative vorticity at both low and middle levels decreased continuously, while the instability index was increased. Smith and Montgomery (2012) also found that, as Gaston was decaying, Convective Available Potential Energy (CAPE) was increasing.

\subsubsection{What caused Gaston to decay?}

Gaston decayed in the face of strong expectations that it would intensify. Davis and Ahijevych (2012) and Smith and Montgomery (2012) hypothesized that Gaston decayed as a result of dry air intrusion. The presence of dry air surrounding Gaston was indeed evident on satellite images. However, we are skeptical of the role of dry air in the initial decay, as our analysis showed that the vertical profile of relative humidity was virtually the same during the first two observations of Gaston (see Fig. 3d). Inspection of the longitudelatitude distribution of the relative humidity and the relative wind at multiple levels on these two consecutive days also does not present evidence of dry air intrusion (Fig. 5 shows the 5 and $7 \mathrm{~km}$ levels). Nor did the Lagrangian analysis of Rutherford and Montgomery (2012) show dry air intrusion between the first two Gaston missions. It appears that the closed circulation of Gaston 1 at both low and middle levels prevented dry air intrusion into the core at this point 
(a) Gaston 1: $3 \mathrm{~km}$

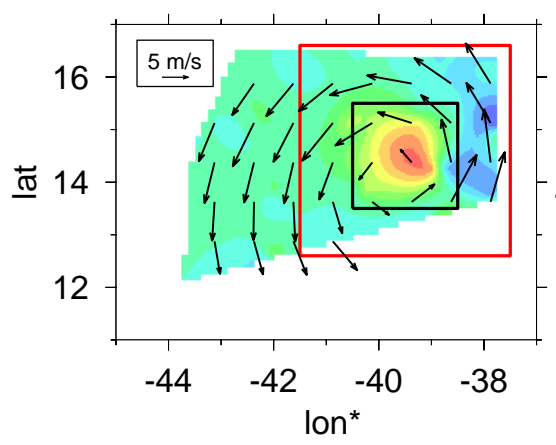

(c) Gaston 1: $6 \mathrm{~km}$

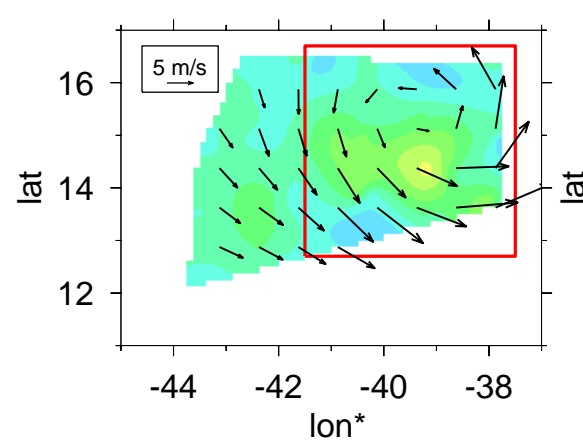

(b) Karl 3: $3 \mathrm{~km}$

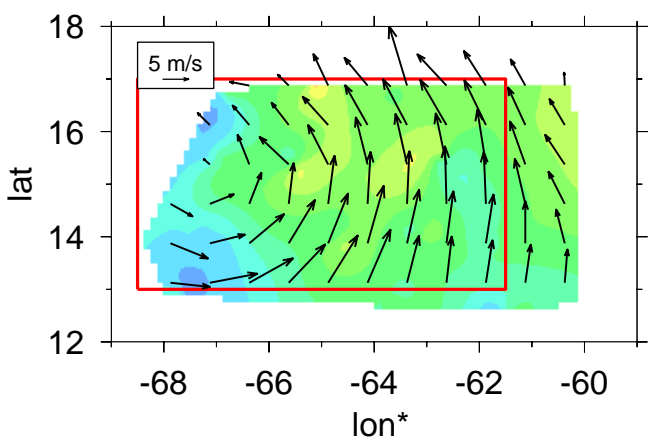

(d) Karl 3: $6 \mathrm{~km}$

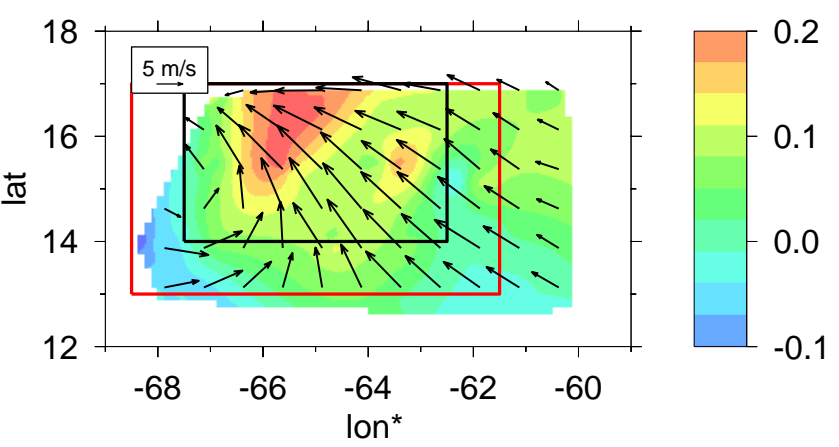

Fig. 6. Vertical mass flux and relative wind at $3 \mathrm{~km}$ and at $6 \mathrm{~km}$ for Gaston 1 (left panels) and Karl 3 (right panels). The units are $\mathrm{kg} \mathrm{m}^{-2} \mathrm{~s}^{-1}$. The red boxes enclose the area selected for analysis, and the black boxes enclose the area of convective activity.

of Gaston's evolution. We do not question the existence of dry air intrusion after Gaston 2. As the above cited papers showed, and as is reflected in our Fig. 5b, the drying of the disturbance started after the second mission into it.

We hypothesize that the severe decrease of the mid-level vortex observed in the period between Gaston 1 and Gaston 2 was a deciding factor for Gaston's failure to re-intensify (see Fig. 3a). Therefore, in this section we explore possible factors that caused this dissipation. We also compare Gaston 1 and Karl 3, as we find that events observed in these two missions were critical to the subsequent success or failure of the respective systems, and the contrast between the two is instructive.

The decrease in the mid-level vorticity from Gaston 1 to Gaston 2 we find to be due to the form of the vertical mass flux profile observed during Gaston 1. The left panels in Fig. 6 show the vertical mass flux at $3 \mathrm{~km}$ and at $6 \mathrm{~km}$ elevation for Gaston 1. Positive vertical mass flux existed near the circulation center in Gaston. However, it was weak in the upper troposphere and it covered a small area. The right panels in Fig. 6 show analogous plots for Karl 3. Convection in Karl 3 was stronger at higher levels and it covered a much larger area compared to that of Gaston 1 . The black boxes in this figure enclose most of the convective activity.
Vertical mass flux profiles for both Gaston 1 and Karl 3, horizontally averaged over the respective black boxes in Fig. 6, are shown in Fig. 7. Consistent with the high instability index previously calculated, Karl 3 had a top-heavy mass flux profile with a maximum vertical mass flux at $10 \mathrm{~km}$. In response to this, Karl exhibited strong mass and vorticity convergence at middle levels and, therefore, intensified mid-level vorticity between Karl 3 and Karl 5. Gaston 1, on the other hand, consistent with the observed low instability index, had a bottom-heavy vertical mass flux profile. It exhibited a maximum at about $3 \mathrm{~km}$, and decreased sharply above that level. The positive vertical gradient of the mass flux in the lowest $3 \mathrm{~km}$ implies mass and vorticity convergence at low levels. As a result, the low-level vortex intensified from Gaston 1 to Gaston 2. However, the negative vertical gradient of the mass flux above $3 \mathrm{~km}$ implies mass divergence at middle levels, and thus vorticity divergence. It seems likely that the decrease in mid-level relative vorticity in this interval weakened the protective pouch at middle levels and allowed dry air intrusion. The dry air sealed the fate of Gaston, as described by Davis and Ahijevych (2012), Smith and Montgomery (2012), and Rutherford and Montgomery (2012). In summary, this entire sequence of events appeared to follow from the weak, bottom-heavy mass flux 
(a) Gaston 1

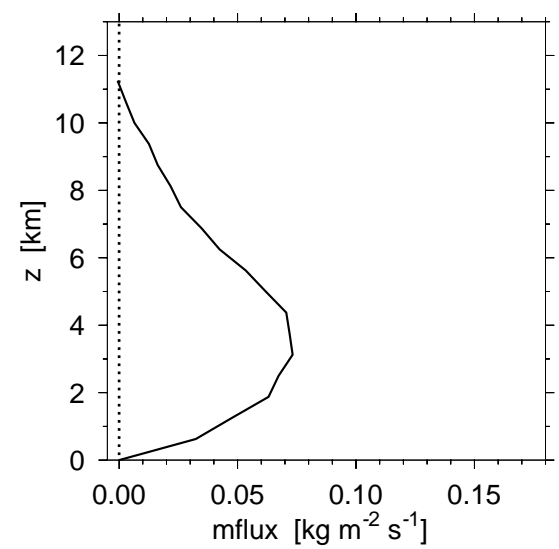

(b) Karl 3

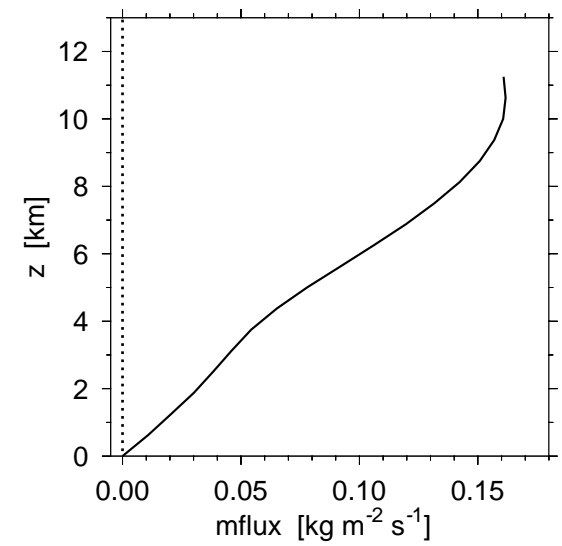

Fig. 7. Vertical mass flux profiles averaged over the black boxes in Fig. 6 for Gaston 1 (left) and Karl 3 (right).

(a) Gaston 1: SST [ $\left.{ }^{\circ} \mathrm{C}\right]$

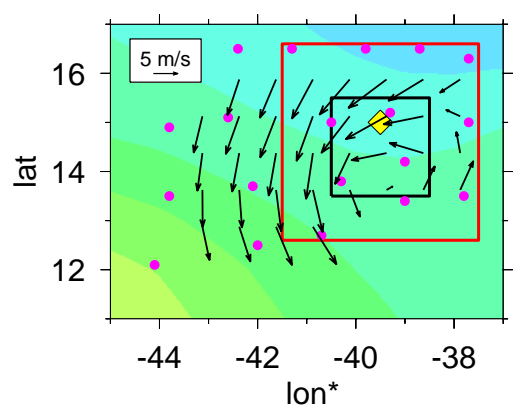

(b) Karl 3: SST [ $\left.{ }^{\circ} \mathrm{C}\right]$

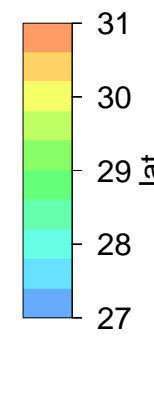

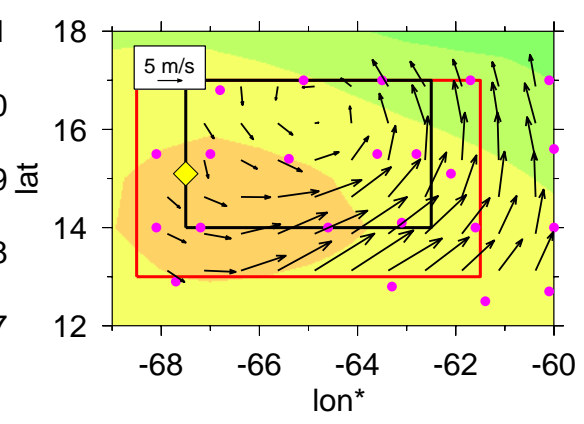

31

30

$-29$

$-28$

27

Fig. 8. Reynolds SST (units are ${ }^{\circ} \mathrm{C}$ ) for Gaston 1 (left) and Karl 3 (right). The vectors represent the relative wind, averaged over the lowest kilometer. The purple dots indicate dropsonde positions, and the yellow diamond marks the circulation center at $5 \mathrm{~km}$.

profile during Gaston 1. Therefore, what is the reason for such convection during Gaston 1 ?

Why was the Gaston 1 mass flux profile so different from that of Karl 3? There are three possibilities for this. First, dry air might have been drawn into the core of Gaston; second, the surface fluxes were likely weaker; and third, buoyancy adjustment from the surroundings may have stabilized the convective column in Gaston. The first possibility is unlikely, as discussed above, due to the robust pouch in Gaston 1.

The underlying ocean provides surface heat fluxes into the disturbance. A warmer ocean provides more energy in the atmosphere. Figure 8a shows the Reynolds SST for Gaston 1 . The overlying vector field is the system-relative wind averaged between 0 and $1 \mathrm{~km}$. The SST below the circulation center is $\sim 28.5^{\circ} \mathrm{C}$ and it decreases northward. Though these SSTs are above the threshold for tropical storm development, they are $2{ }^{\circ} \mathrm{C}$ cooler then the corresponding SSTs for Karl 3 (Fig. 8b). The stated threshold does not ensure development and $2^{\circ} \mathrm{C}$ difference in SST can make a large difference in surface fluxes. Furthermore, in recent years studies have found that the underlying upper ocean heat con- tent, rather then the SST, is a better representative of the ocean's input into the disturbance. The higher the heat content, the higher the transport from the ocean to the atmosphere (Wada and Usui, 2007). Figure 9 is taken from National Oceanic and Atmospheric Administration's (NOAA) website and it shows the Tropical Cyclone Heat Potential (TCHP), a variable representative of the ocean heat content. Roughly estimated from the figure, the average TCHP for Gaston 1 is less then $40 \mathrm{~kJ} \mathrm{~cm}^{-2}$. For Karl 3 the average is around $80 \mathrm{~kJ} \mathrm{~cm}^{-2}$, with largest values of $100 \mathrm{~kJ} \mathrm{~cm}^{-2}$, approximately collocated with the circulation center of the disturbance. This large difference in TCHP is potentially an important factor for the observed differences in the mass flux profiles of Gaston 1 and Karl 3.

Now we explore moist entropy and buoyancy of ascending surface parcels in Gaston 1 and Karl 3 (see Appendix A for buoyancy calculation). We calculated these variables as averages over dropsondes from the respective disturbance, within the area selected for analysis. We averaged separately over the dropsondes within the respective black boxes (see Fig. 8) that enclose most of the convectively active regions, and over 

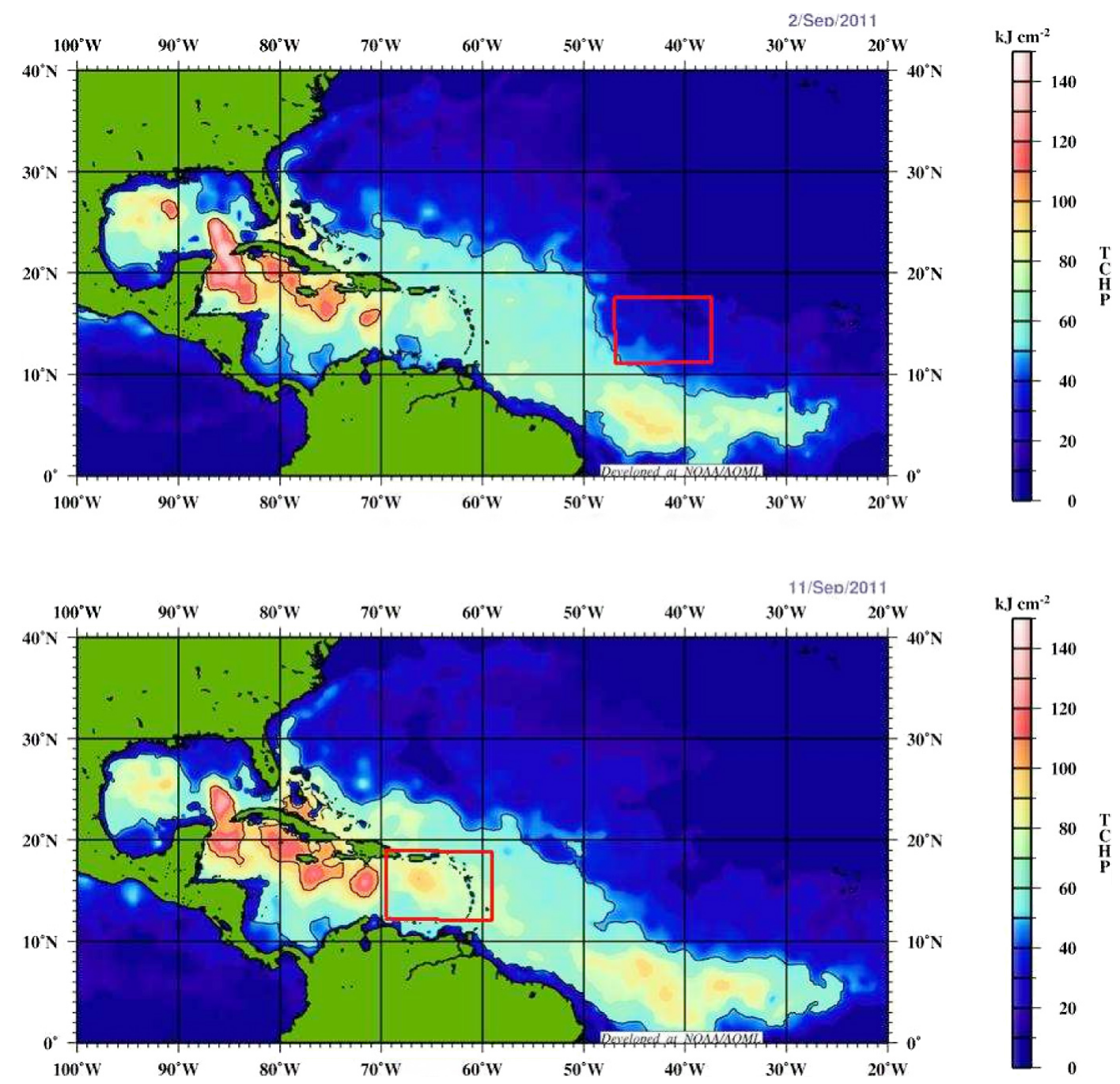

Fig. 9. TCHP for Gaston 1 (top panel) and Karl 3 (bottom panel). The red boxes mark approximately the respective observational areas. The graphics are taken from http://www.aoml.noaa.gov/phod/cyclone/data.

the dropsondes outside these regions, but still within the selected area for analysis. The results are shown in Fig. 10. The saturated moist entropy for Gaston 1 indicates a temperature inversion. The inversion is especially evident outside the black box, even though it is present within the black box as well (Fig. 10a, red dashed red line and black dashed line, respectively). Within the convectively active region the buoyancy profile does not show evidence of an inversion (Fig. 10b), most likely because convection locally destroys the inversion. Outside the black box in Gaston 1, both saturated moist entropy and buoyancy indicate a strong trade wind inversion and overall a much smaller magnitude of buoyancy. In contrast, the corresponding entropy and buoyancy vertical profiles of Karl 3 (Fig. $10 \mathrm{c}$ and d) show no evidence of an inversion inside or outside of the convectively active region, which is consistent with the high values of the underlying SSTs. There is a notable difference between the buoyancy profiles of Gaston 1 and Karl 3. For Gaston 1 there is a big difference in buoyancy within and outside the convectively active region, whereas for Karl 3, this difference is much smaller. Thus, the larger environment (outside the box) for Karl 3 is conducive to convection, whereas for Gaston 1 it is very unfavorable. We hypothesize that in Gaston 1 convection is modifying buoyancy locally, and thus the inversion is reduced in the region of convective activity, while at the same time the trade wind dynamics are reinforcing the temperature inversion, which tends to suppress convection.

In summary, our analyses demonstrate a hostile environment for convection in Gaston 1 in the form of a strong trade wind inversion. We hypothesize that the inversion, originating from the relatively low SSTs, together with the low ocean heat content, was the main factor that suppressed convection. The corresponding observed mass flux profile then resulted in a sharp decrease of the mid-level vorticity from Gaston 1 
(a) Gaston 1

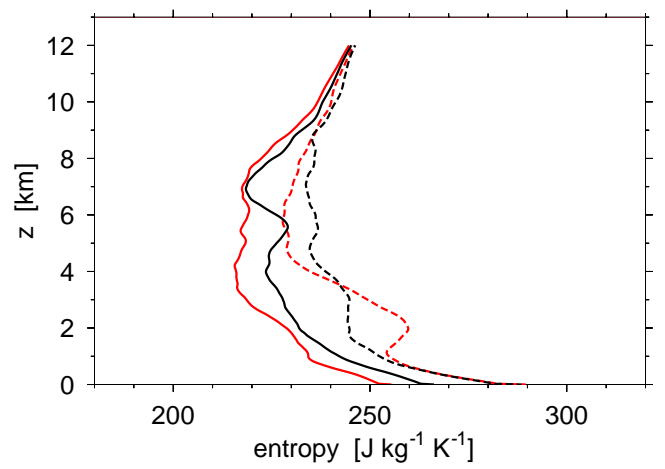

(c) Karl 3

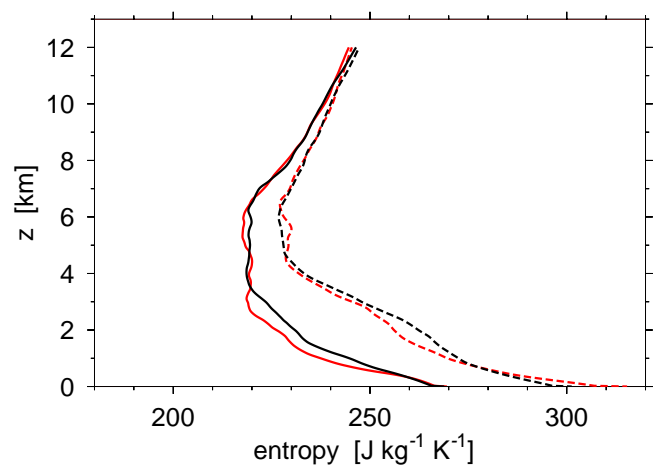

(b) Gaston 1

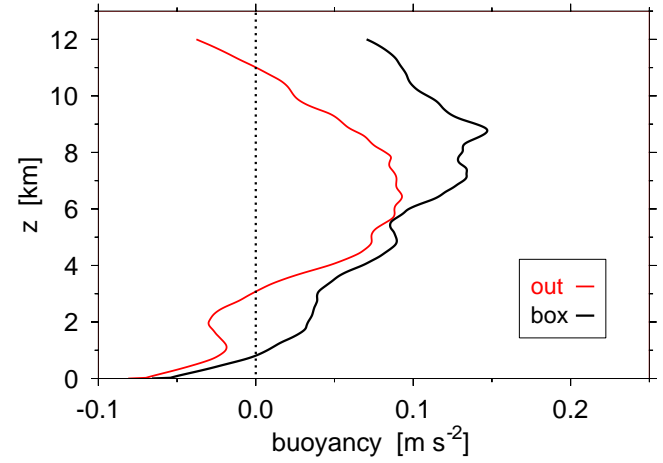

(d) Karl 3

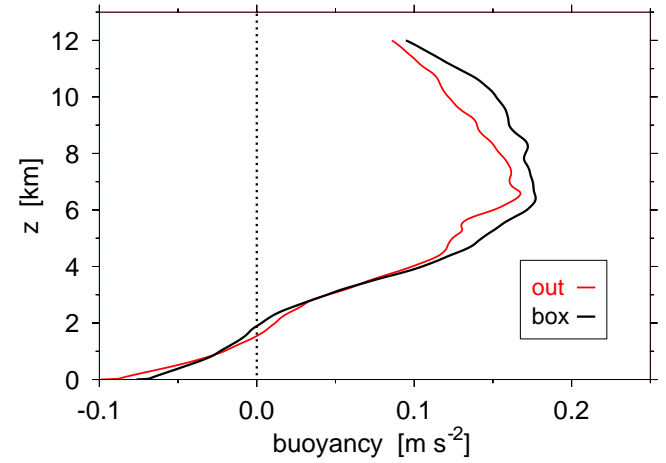

Fig. 10. Vertical profiles of (a) moist entropy (solid lines) and saturated moist entropy (dashed lines) for Gaston 1, (b) buoyancy for Gaston 1 , (c) moist entropy (solid lines) and saturated moist entropy (dashed lines) for Karl 3, and (d) buoyancy for Karl 3. The black lines represent averages over the dropsondes inside the respective black boxes in Fig. 8, and the red lines represent averages over the dropsondes enclosed with the respective red box, but outside of the black boxes in Fig. 8 .

to Gaston 2. This led to the collapse of the pouch at midlevels and the subsequent intrusion of dry air into Gaston's core, resulting in its failure to develop. Low relative humidity above $6 \mathrm{~km}$ may have been an additional factor, but this effect was likely weak due to the low saturation mixing ratio and hence low saturation deficit at high elevations. Dry air surrounded Gaston at middle levels, but initially appeared to be unable to penetrate to the core due to the protective pouch produced by the mid-level vortex, thus limiting its direct effect on convection during Gaston 1.

\section{Conclusions}

This paper uses observations from three field programs to explore tropical storm formation. Possible correlations between dynamics and thermodynamics were explored in Sect. 5.1 and detailed analyses of two disturbances observed during PREDICT were presented in section 5.2. The vertical profiles of the various variables presented represent areal averages. Because of airspace restrictions some of the observa- tional domains were not centered over the target disturbance. Thus, the vertical profiles most likely differ from what they would be if averaged over the exact area that the respective disturbances cover. Given the small timescales and horizontal scales of convection versus the larger timescales and horizontal scales of mesoscale vorticity, the mean vertical vorticity profile is probably much less sensitive to the domain selection than the mass flux profile. Therefore, it is more robust in representing the big picture of the disturbance behavior, unless the circulation center is entirely outside of the observational domain. Missions where the latter was the case do not enter the analyses in Sect. 5.2. Davis and Ahijevych (2012) have used other data sources in addition to the dropsonde data and have used a different treatment in calculating the vertical circulation profiles of their analyses of Gaston, Karl and Matthew. The results they obtained do not differ qualitatively from the results presented in this paper which reinforces our analysis.

The results presented stress the importance of the midlevel vortex. We found that the development of a strong 
mid-level vortex preceded low-level vortex intensification in the developing case, and divergence of mid-level vorticity preceded weakening of the low-level vortex in the decaying case. The important question that we attempt to answer in this paper is how the mid-level vortex communicates with the dynamics at low levels. The evolution of the dynamics and thermodynamics of Karl and Gaston, the trends in the scatter plots, and the previous theoretical work by Raymond and Sessions (2007), support the following theory on the mechanisms by which the mid-level vortex promotes low-level spin up: by virtue of thermal wind balance, the mesoscale midlevel vortex maintains a more stable atmosphere. We saw from observations that stronger mid-level vorticity was indeed associated with a lower instability index (Fig. 1a). The more stable atmosphere is conducive to moist convection that produces a bottom-heavy mass flux profile, meaning that it exhibits the largest positive vertical gradient in a shallow layer adjacent to the sea surface. Mass continuity then dictates horizontal mass convergence at low levels, where most of the water vapor is contained. Mass convergence near the surface means water vapor convergence and low-level vorticity convergence. Hence the negative correlations between the instability index and the saturation fraction (Fig. 1b) and between the instability index and the low-level vorticity tendency (Fig. 1c). If the mid-level vorticity exists long enough to keep this chain of events going, the low-level wind speed will eventually reach the tropical storm threshold.

The theory presented here is in agreement with the marsupial paradigm that the low-level vortex intensifies as a result of the aggregation of vorticity produced by deep convection. Our theory focuses on the vertical mass flux that most efficiently leads to aggregation of vorticity. From observations, we found that it is a mass flux with the strongest positive vertical gradient in a shallow layer near the surface. Our theory says that the mid-level vortex is necessary to maintain thermodynamic stratification on the mesoscale that is conducive to such convection. We believe that the protective pouch, defined by the marsupial paradigm, also plays an important role in supporting convection in that it blocks the ingestion of dry environmental air into the convective region. Furthermore, the mid-level vortex is instrumental in creating the mid-level pouch, and, as Gaston illustrates, its absence facilitates the ingestion of environmental air.

Karl was a disturbance that closely followed our theory. It was observed in the 4-day period before it become a tropical storm. It started with a low-level cyclonic vortex and a weaker mid-level vortex, but it did not start intensifying until it developed a strong mid-level vortex. The development of the mid-level vortex followed as a result of the strong, top-heavy vertical mass flux profile that was observed during Karl 3. This profile is consistent with the high instability index that existed at that time. Karl 5 exhibited strong midlevel vorticity and a low instability index which resulted in low-level vortex spin up between Karl 5 and Karl 6.
Gaston was first observed only a couple of hours after it decayed from a tropical storm to a tropical depression. During this period, Gaston still had a strong mid-level vortex, which had weakened significantly by the second mission into this disturbance. We believe that this was the crucial element in Gaston's decay. We further hypothesize that convection was suppressed by a strong trade wind inversion. Convection at the time of the observation was concentrated in a small area near the $5 \mathrm{~km}$ circulation center. It produced a bottomheavy vertical profile of the convective mass flux, which decreased with altitude in the middle troposphere. Though the bottom-heavy mass flux profile produced a transient intensification of the low-level vortex, the negative vertical gradient of the mass flux above $3 \mathrm{~km}$ was responsible for the negative vorticity tendency in the middle levels and therefore the decrease in mid-level vorticity by the following day. The divergence of the mid-level vorticity exposed Gaston to the ingestion of dry environmental air, which sealed its fate.

Acknowledgements. We thank the dedicated staff of the NSF/NCAR G-V operation for their excellent work. Data provided by NCAR/EOL under sponsorship of the National Science Foundation. This work was supported by National Science Foundation grants 0851663 and 1021049, and the Office of Naval Research grant N000140810241.

Edited by: T. J. Dunkerton

\section{References}

Bister, M. and Emanuel, K. A.: The genesis of hurricane Guillermo: TEXMEX analyses and a modeling study, Mon. Weather Rev., 125, 2662-2682, 1997.

Braun, S. A., Kakar, R., Zipser, E., Heymsfield, G., Albers, C., Brown, S., Durden, S. L., Guimond, S., Halverson, J., Heymsfield, A., Ismail, S., Lambrigsten, B., Miller, T., Tanelli, S., Thomas, J., and Zawislak, J.: NASA's Genesis and Rapid Intensification Processes (GRIP) field experiment, B. Am. Meteor. Soc., 94, 345-363, 2012.

Davis, C. A., and Ahijevych, D. A.: Mesoscale structural evolution of three tropical weather systems observed during PREDICT, J. Atmos. Sci., 69, 1284-1305, 2012.

Dunkerton, T. J., Montgomery, M. T., and Wang, Z.: Tropical cyclogenesis in a tropical wave critical layer: easterly waves, Atmos. Chem. Phys., 9, 5587-5646, doi:10.5194/acp-9-5587-2009, 2009.

Gray, W. M.: Global view of the origin of tropical disturbances and storms, Mon. Weather Rev., 96, 3380-3403, 1968.

Haynes, P., and McIntyre, M.: On the evolution of vorticity and potential vorticity in the presence of diabatic heating and fractional or other forces. J. Atmos. Sci., 44, 828-841, 1987.

Harr, P. A., and Elsberry, R. L.: Transformation of a large monsoon depression to a tropical storm during TCM-93, Mon. Weather Rev., 124, 2625-2643, 1996.

López Carrillo, C. and Raymond, D. J.: Retrieval of threedimensional wind fields from Doppler radar data using an ef- 
ficient two-step approach, Atmos. Meas. Tech., 4, 2717-2733, doi:10.5194/amt-4-2717-2011, 2011.

McBride, J. L., and Zehr, R.: Observational analysis of tropical cyclone formation. Part II: Comparison of non-developing versus developing systems, J. Atmos. Sci., 38, 1132-1151, 1981.

Montgomery, M. T., Davis, C., Dunkerton, T., Wang, Z., Velden, C., Torn, R., Majumdar, S. J., Zhang, F., Smith, R. K., Bosart, L., Bell, M. M., Haase, J. S., Heymsfield, A., Jensen, J., Campos, T., and Boothe, M. A.: The Pre-Depression Investigation of Cloudsystems in the Tropics (PREDICT) experiment, B. Am. Meteor. Soc., 93, 153-172, 2012.

Montgomery, M. T. and Smith, R. K.: The genesis of Typhoon Nuri as observed during the Tropical Cyclone Structure 2008 (TCS08) field experiment - Part 2: Observations of the convective environment, Atmos. Chem. Phys., 12, 4001-4009, doi:10.5194/acp-124001-2012, 2012.

Nolan, D. S.: What is the trigger for tropical cyclogenesys?, Aust. Met. Mag., 56, 241-266, 2007.

Raymond, D. J.: Balanced thermal structure of an intensifying tropical cyclone, Tellus-A, 64, 19181, doi:10.3402/tellusa.v64i0.19181, 2012.

Raymond, D. J. and López Carrillo, C.: The vorticity budget of developing typhoon Nuri (2008), Atmos. Chem. Phys., 11, 147163, doi:10.5194/acp-11-147-2011, 2011.
Raymond, D. J. and Sessions, S. L.: Evolution of convection during tropical cyclogenesis, Geophys. Res. Lett., 34, L06811, doi:10.1029/2006GL028607, 2007.

Raymond, D. J., Sessions, S. L., and López Carrillo, C.: Thermodynamics of tropical cyclogenesis in the Northwest Pacific, J. Geophys. Res., 116, D18101, doi:10.1029/2011JD015624, 2011.

Reasor, P. D., Montgomery, M. T., and Bosart, L. F.:Mesoscale observations of the genesis of Hurricane Dolly (1996), J. Atmos. Sci., 62, 3151-3171, 2005.

Rutherford, B. and Montgomery, M. T.: A Lagrangian analysis of a developing and non-developing disturbance observed during the PREDICT experiment, Atmos. Chem. Phys., 12, 11355-11381, doi:10.5194/acp-12-11355-2012, 2012.

Smith, R. K. and Montgomery, M. T.: Observations of the convective environment in developing and non-developing tropical disturbances, Q. J. Roy. Meteorol. Soc., 138, 1721-1739, 2012.

Wada, A. and Usui, N.: Importance of tropical cyclone heat potential for tropical cyclone intensity and intensification in the western north pacific, J. Oceanogr., 63, 427-447, 2007.

Wang, Z.: Thermodynamic Aspects of Tropical Cyclone Formation, J. Atmos. Sci., 69, 2433-2451, 2012.

Wissmeier, U. and Smith, R. K.: Tropical cyclone convection: the effects of ambient vertical vorticity, Q. J. Roy. Meteor. Soc., 137, 845-857, 2011. 


\section{Appendix A}

\section{Buoyancy calculation}

We calculate the buoyancy as follows:

$b=-g\left(T_{\mathrm{v}}-T_{\mathrm{vp}}\right) / T_{\mathrm{v}}$.

$T_{\mathrm{V}}$ is the environmental virtual temperature,

$T_{\mathrm{v}}=T(1+0.00061 r)$,

and $T_{\mathrm{vp}}$ is the parcel virtual temperature,

$T_{\mathrm{vp}}=T_{\mathrm{p}}\left(1+0.00061 r_{\mathrm{p}}\right)$.

$T_{\mathrm{p}}$ is the parcel temperature at each level and it is calculated as the environmental temperature plus a temperature perturbation, $\delta T$, of the parcel at each level. The latter is estimated by using the approximate formulas (7) and (8) from RSL11. These are $\delta T=\delta s^{*} /\left(\partial s^{*} / \partial T\right)_{\mathrm{p}} \approx \delta s^{*} /\left(3.7+0.00064 r^{*}\right)$,

where we calculate $\delta s^{*}$ as

$\delta s^{*}=s^{*}-s_{0}$.

Here $s_{0}$ is the specific moist entropy averaged over the lowest kilometer and $s^{*}$ is the saturated moist entropy of the environment. The variable $r_{\mathrm{p}}=r_{\mathrm{p}}(z)$ in Eq. (A3) is the mixing ratio of the parcel, which we calculate from the pressure and $T_{\mathrm{p}}$, on the assumption that the parcel is saturated. We use the formula

$r_{\mathrm{p}}=0.622 \frac{e_{\mathrm{s}}\left(T_{\mathrm{p}}\right)}{p-e_{\mathrm{s}}\left(T_{\mathrm{p}}\right)}$,

where $e_{\mathrm{S}}$ is the saturation vapor pressure calculated from the Clausius-Clapyron equation. The water vapor mixing ratios in the above equations, $r_{\mathrm{p}}, r^{*}$, and $r$, have units of $\mathrm{kg} \mathrm{kg}^{-1}$. 